\title{
Development and evaluation of macrophage targeted multidrug therapy against visceral leishmaniasis
}

\author{
Prachi Sharma ${ }^{1}$, Swati Gupta ${ }^{2 *}$ \\ ${ }^{1}$ School of Pharmaceutical Science, Apeejay Stya University, Gurgaon, India \\ ${ }^{2}$ Department of Pharmaceutics, B. S. Anangpuria Institute of Pharmacy, Faridabad, Haryana, India
}

Received: Feb 13, 2017, Revised: Feb 27, 2017, Accepted: Apr 20, 2017

\begin{abstract}
In this study, we fabricated PCL-nanoparticles by encapsulating dual drugs as amphotericin B and doxorubicin via doubleemulsion solvent evaporation method also incorporated with ligand-lectin for targeting the infested macrophage cells and prove importance against VL. Different independent processing parameters were assessed systematically to enhance the incorporation of the dual agents with different properties (AmB and DOX, hydrophobic \& hydrophilic molecule, respectively) into PCL-NPs and control particle size. Approaches investigated for the enhancement of drug entrapment efficiencies and smaller particle size included the influence of the drug content, polymer content, sonication time etc. The mean particle size and zeta potential of PCLNPs were $236.7 \pm 0.04 \mathrm{~nm}$ in diameter and $-9.11 \pm 3.46 \mathrm{mV}$, respectively. The entrapment efficiencies of AmB and DOX were $82.1 \pm 1.39$ and $75.20 \pm 0.14 \%$, respectively. Antileishmanial activities of the formulations and various combination approaches were assessed using macrophage-specific ligand-lectin. The prepared plain and lectin coated PCL-NPs based systems showed remarkable potential for passive and active intra macrophage targeting, respectively and the approach could be a successful alternative to the currently available drug regimens against VL. Multidrug resistance can be improved by combination delivery of encapsulated anti VL drugs. Thus, the co-encapsulation of AmB and DOX should reduce side effects of both drugs while increasing efficacy.
\end{abstract}

Keywords: PCL-Nanoparticles, amphotericin B, doxorubicin, lectin, macrophages, macrophage targeting

Pharm Biomed Res 2017; 3(1): 14-21

\section{Introduction}

Visceral leishmaniasis (VL) also known as kala-azar caused by "Leishmania donovani" and transmitted by the sandfly, "Phlebotomus argentipes" is the most severe form of leishmaniasis and is almost fatal, if left untreated. It results from infection of the macrophages of the liver, spleen and bone marrow. The symptoms of conventional disease are fever, weight loss, hepatosplenomegaly and pancytopenia. It occurs in more than 80 countries in Africa, Asia, Southern Europe and South America. Approximately 500,000 new cases of VL occur worldwide each year. India alone may contribute as much as $40-50 \%$ of these with $90 \%$ occur in the state of Bihar (1).

Despite the accessibility of several drugs, currently existing therapies for VL are very toxic and also started showing emergence of drug-resistant parasitic strains. For instance, pentavalent antimonials (sodium stibogluconate) and pentamidine were potent drugs used against VL; but there is emerging evidence that the rates of response are declining due to the appearance of resistance, relapses due to the emergence of several unresponsive strains of the leishmanial parasite and toxicity (hypotension, reversible hypoglycemia, and nephrotoxicity) (2). Miltefosine is a relatively new anti-VL drug which holds significant potential in treating VL along with other first-line therapeutics (3); however, primary mechanism is quite uncertain, and possible impairment of lipid metabolism occurs. Despite all research initiatives and preclinical studies, effective therapy for VL still remains challenge holding direct negative implications with respect to better drug targeting and overcoming drug resistance (3). Amphotericin B $(\mathrm{AmB})$ is used as a prototype leishmanicidal drug due to its excellent efficacy and its use results in fewer treatment failures and relapses $(4,5)$. However, the important side effects, mainly nephrotoxicity, produced by this drug at therapeutic doses have often led to its rejection as a first-choice treatment. Counter intuitively, doxorubicin (DOX), an anthracycline antibiotic widely used anticancer agents (6), was reported to be equally effective (in comparison to amphotericin) as an anti-VL agent based on the studies conducted by Sett et al. and other researchers (7). Although highly potent, activity of DOX has been restricted by its fatal toxicity, cost-effectiveness, dose limited cardiotoxicity and myelosuppression $(8,9)$. The problem requires that the drug should be targeted to the macrophages in such a way that the interaction of the free drug 
with non-target tissues could be minimized (10). The possibility of improving the plasma half-life of the drugpolymer conjugate, with maximum accumulation in the target site, can be achieved through encapsulation of the drug in a long circulating carrier such as liposomes or nanoparticles. Out of these carriers, the biodegradable Nano-particulate systems (NPs) have recently received considerable attention as potential drug delivery systems (11).

The aim of the present work is to formulate poly- $€$ caprolactone-nanoparticles (PCL-NPs) with a view towards vectoring combination of drugs (AmB and DOX) to RES incorporated with macrophage targeted ligand- lectin and generated an excellent tool that can circumvent major drug related issues in the treatment of VL. One such approach to increase the efficacy and to reduce the dose related toxicity of these drugs is to target the drug molecule to the phagolysosomes of the MPS where the leishmania parasites reside (12). These nanoparticles are suitable for passive and active targeting of drugs to MPS and can result in higher concentrations of drug in liver and spleen on parenteral administration (9). They can be passively taken up from the blood stream by the macrophages of the liver and spleen after intravenous or intracardiac administration because of their colloidal nature. The PCL-NPs are stable formulations that could be an economically attractive substitute of the already licensed AmB and DOX formulations. Concurrently, ligand mediated active targeting to the macrophages with the proposed PCL-NPs would considerably increase the rate and extent of macrophage accretion of drug which may further decrease the dose size and dose frequency of already approved formulations.

\section{Materials and methods \\ Materials}

Amphotericin B (AmB) was procured as a gift sample from Lifecare Innovations, Gurugram, India. Doxorubicin Hydrochloride (DOX) was received as gift sample from Sun Pharmaceuticals Industries Limited, India. Poly-€-caprolactone (PCL) (85,000 Da), lectin (WGA), poly-vinyl alcohol (PVA, Av. MW 30,000-70,000), Eagle's medium and Locke's solution (MWCO: 3500) were purchased from Sigma Chemical Co. USA. All other chemicals used were of analytical reagent grade and without further purification.

\section{Preparation of PCL-nanoparticles}

PCL-nanoparticles (PCL-NPs) containing AmB and DOX were prepared by using double emulsion method with slight modification as per our laboratory set up (13). In a small plastic vial $58.9 \mathrm{mg}$ PCL was mixed with $1 \mathrm{ml}$ of chloroform and added in previously extracted DOX under magnetic stirring (Remi, Mumbai, India). After which DOX solution was added in $0.1 \% \mathrm{w} / \mathrm{v}$ PVA solution. In a separate plastic vial $0.5 \mathrm{mg}$ AmB was dissolved in $0.2 \mathrm{~mL}$ dimethyl sulphoxide (DMSO) and injected into PVA solution and resulted emulsion was stirred at room temperature under probe sonicator (Sonics, Vibracell) at 10,000 rpm, for $15 \mathrm{~min}$. The resultant PCL-NPs were separated by ultracentrifugation at 16,000 rpm (Sigma 3K- 18 refrigerated centrifuge, Germany) for $30 \mathrm{~min}$ and washed with distilled water at least three times (14).

\section{Optimization of PCL-nanoparticles}

Optimization of PCL-Nanoparticles was carried out by varying different parameters such as drug content, polymer content and sonication time. For optimization of drug content (AmB and DOX) to polymer content, polymer content $(50 \mathrm{mg})$ and sonication time (5 min) were kept constant while $\mathrm{AmB}$ and DOX content was varied at different weight levels, i.e. (2, 3, 4, $5 \mathrm{mg}$ for $\mathrm{AmB}$ and 5, 10, 15, and $20 \mathrm{mg}$ for DOX) in different formulations (Table 1) for determining optimum AmB and DOX content (15). Average particle size of different formulations was determined by particle size analyzer (Delsa Nano C, Beckman Coulter, USA) and percent drug entrapment in different formulations was also determined as described previously (16). PCL-NPs with optimum AmB and DOX to polymer content were optimized for optimum sonication time in terms of average particle size. PCL content, drug content (AmB and DOX) to polymer content (optimized), and \%w/v PVA concentration were kept constant while sonication time was varied (i.e. 5, 10, 12, 15, $20 \mathrm{~min}$ ) for different formulations. PCL-NPs were evaluated for average particle size by photon correlation spectroscopy using Zetasizer Nanoseries (Nano-ZS, Malvern Inst. Ltd. UK) using a flow-through cell and percent drug entrapment in different formulations was also determined. We have found maximum drug encapsulation efficiencies with optimum sonication time at $15 \mathrm{~min}$. Formulation with optimum drug content and sonication time were optimized for optimum polymer content (PCL) in terms of percent drug entrapment. The PCL-NPs with fixed drug content, sonication time with different polymer content (PCL) (i.e 50, 100, 150, $200 \mathrm{mg}$ ) were prepared (12). Drug content and sonication time however were kept constant at its optimum level. Increasing the amount of PCL from 50$200 \mathrm{mg}$ caused NPs size shift toward a higher size range from 236.7 to $572 \mathrm{~nm}$, and entrapment efficacy of AmB and DOX was decreased from to $82.1 \pm 1.39$ to $55.76 \%$ and $75.20 \pm$ 0.14 to $32 \%$ respectively (10). PCL-NPs were evaluated for percent drug entrapment and percent haemolysis. Percent haemolysis was determined by the method reported in 
literature (16). The optimization method for PCL-NPs was done to produce NPs of small size with high entrapment efficiency (Figure 1).

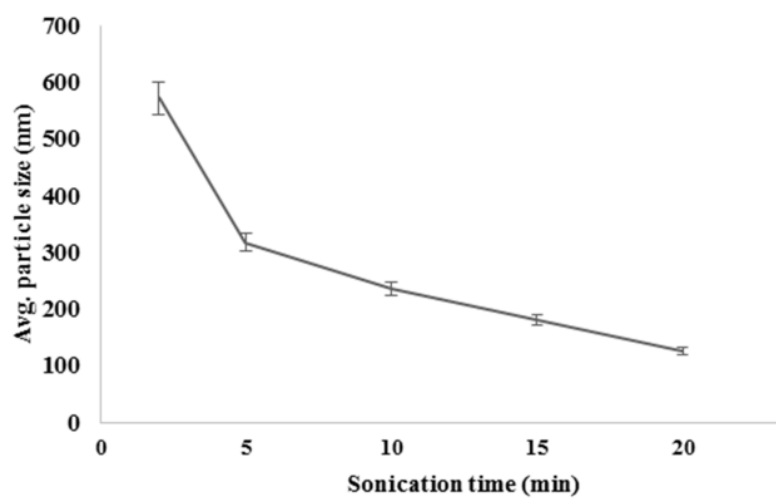

Figure 1 Optimization of sonication time in terms of average particle size total polymer used (PCL) - $50 \mathrm{mg}, \mathrm{AmB}-5 \mathrm{mg}$, DOX- $10 \mathrm{mg}$

To incorporate the surface of PCL-NPs with lectin, a modified emulsion-solvent evaporation method $\left(\mathrm{w}_{1} / \mathrm{o} / \mathrm{w}_{2}\right)$ was performed with slight modification as per our laboratory setup (17). For coating, $1 \mathrm{~mL}$ PCL-NPs was incubated and gently stirred with $3 \mathrm{~mL}$ lectin dispersion at room temperature. The hardened PCL-NPs were washed twice with distilled water by ultracentrifugation (Sigma $3 \mathrm{~K}-18$ refrigerated centrifuge, Germany, 40,000 g, $1 \mathrm{~h}, 4{ }^{\circ} \mathrm{C}$ ), the whole process being repeated thrice to ensure complete removal. The two process variables, total lectin ratio to zeta potential and incubation time required for coating were optimized by measuring the change in zeta potential of the dispersion. For optimization of zeta potential: lectin ratio and zeta potential: incubation time for the effective coating, negatively charged PCL-NPs were incubated with varying amounts of lectin, i.e. w/w ratios based on polymer weight $(0: 10,1: 15,1: 20(\mathrm{w} / \mathrm{w})$ ratio) for a fixed time period of $6 \mathrm{hr}$ and zeta potential was measured (Delsa Nano C, Beckman Coulter, USA). The optimum ratio was determined at which no significant change in zeta potential was recorded on further increasing the lectin. Similarly for the optimization of incubation time, the formulations were incubated with optimum lectin content for different time periods $(0,1,2,3,4,5,6,7 \mathrm{~h})$ and change in zeta potential was measured (18).

\section{In-vitro characterization}

Developed PCL-NPs were characterised before and after surface ligand anchoring. The size distribution of PCL-NPs was determined in bi-distilled water by photon correlation spectroscopy (PCS) using a particle size analyser (Brookhaven Instruments Corps). For the determination, $1 \mathrm{~mL}$ of NPs was dispersed in $5 \mathrm{~mL}$ of distilled water and sonicated for about 1 $\mathrm{hr}$. The analyses were performed at a scattering angle of $90^{\circ} \mathrm{C}$ and a temperature of $25^{\circ} \mathrm{C}$. Formulations were evaluated for their shape and morphology by transmission electron microscopy (TEM) (Hitachi 7500, Japan). Phosphotungstic acid (1\%) was used as a negative stain. Carbon coated samples were placed over a copper grid and subjected to TEM analysis. In vitro drug release was performed using dialysis membrane diffusion technique. Briefly, $1 \mathrm{~mL}$ of encapsulated formulation was suspended in $1 \mathrm{~mL}$ of phosphate buffer solution (PBS, pH 7.4) in a dialysis bag and dialyzed against $250 \mathrm{~mL}$ PBS with $0.5 \%$ Tween 80 contained in dissolution apparatus (DISSO 2000, Labindia, India), thermostated at $37 \pm 1{ }^{\circ} \mathrm{C}$ with moderate shaking at $100 \mathrm{rpm}$. At specific time intervals, a definite volume $(1 \mathrm{~mL})$ of the release medium was withdrawn and replenished with fresh PBS and analyzed for AmB and DOX amount using validated HPLC method each for both drugs (AmB and DOX). Each measurement was performed in triplicate and reported as their average (5).

In order to quantify the drug entrapment efficiency encapsulated amount, AmB and DOX was extracted from the PCL-NPs using dimethyl sulfoxide, diluted with methanol and analyzed using HPLC. The HPLC (LC-10ATvp, Shimadzu, Tokyo, Japan) was equipped with a Lichrosphere reversephase C18 column $(250 \times 4 \mathrm{~mm}, 5 \mu \mathrm{m}$; Merck, Darmstadt, Germany). Acetonitrile with $\mathrm{KH}_{2} \mathrm{PO}_{4}$ buffer ( $\mathrm{pH} 3.5$, adjusted with orthophosphoric acid), (60:40, v/v) was employed as mobile phase at $1.0 \mathrm{ml} / \mathrm{min}$ flow rate and column effluent was detected with a UV detector at 405 and $235 \mathrm{~nm}$. Results are expressed as AmB and DOX actual loading (drug amount encapsulated per $100 \mathrm{mg}$ of PCL-NPs) and encapsulation efficiency (EE) (ratio between drug amount entrapped in the PCL-NPs and that added during PCL-NPs preparation) \pm SD of values collected from three different batches (10).

\section{Ex-vivo stability}

The Ex-vivo studies were performed by observing drug leaching and change in particle size following incubation of PCL-NPs with freshly pooled rat serum at $37 \pm 1{ }^{\circ} \mathrm{C}$. The drug content of the PCL-NPs was determined by the method described previously with slight modifications (16). PCL-NPs formulations $(1 \mathrm{~mL})$ were incubated with $2 \mathrm{ml}$ serum at $37 \pm$ $1^{\circ} \mathrm{C}$ for $1,2,4,6$ and $24 \mathrm{hrs}$. After specified time intervals, suspensions were centrifuged at 20,000 rpm for $15 \mathrm{~min}$ and supernatant was filtered through $0.22 \mu \mathrm{m}$ membrane filter. The filtrate was analyzed for drug content by reverse phase high performance liquid chromatography (HPLC) method as described previously (10). The particle size of PCL-NPs formulations was determined after $24 \mathrm{hrs}$ incubation of the 
formulations with the serum using particle size analyzer (CILAS, 1064, Marcoussis, France) (5).

In vitro phagocytic uptake studies in macrophage cell line Adherent mouse macrophage cell line J774A.1 was used for in vitro activity against intracellular amastigotes in macrophages, maintained in Dulbecco's modified Eagle's medium (Sigma, USA) supplemented with $10 \%$ heat-inactivated foetal bovine serum, $100 \mathrm{U} / \mathrm{mL}$ penicillin and $100 \mathrm{mg} / \mathrm{mL}$ streptomycin at $37{ }^{\circ} \mathrm{C}$ in $5 \% \mathrm{CO}_{2}$ in humidified atmosphere (2). Macrophages $\left(1 \times 10^{5}\right.$ cells/well) in 16-well chamber slides (Nunc, Naperville, IL, USA) were infected with promastigotes (L. donovani, Dd8) at multiplicity of infection of 10:1 (parasites: macrophage) and incubated at $37.8{ }^{\circ} \mathrm{C}$ in $5 \% \mathrm{CO}_{2}$ for $12 \mathrm{hrs}$ after which chamber slides were washed thrice with PBS (pH 7.2) to remove non-phagocytosed promastigotes and finally supplemented with medium. Different concentrations $(0.03$, $0.08,0.13$ and $0.2 \mathrm{mg} / \mathrm{ml}$ ) of $100 \mathrm{~mL}$ of free PCL-NPs, PCLNPs (AmB and DOX), lectin-PCL-NPs in RPMI-1640 medium were added to wells in triplicate. The untreated infected macrophages were used as control. Formulations were then removed by washing after $3 \mathrm{hrs}$ and macrophages were placed in medium for an additional $20 \mathrm{hrs}$ and then examined for intracellular amastigotes under oil immersion objective of light microscope after methanol fixing and Giemsa staining of the slides. At least 100 macrophage nuclei were counted per well for calculating the percentage infected macrophages anc number of amastigotes per 100 macrophages. After washing cells were fixed in $10 \% \mathrm{w} / \mathrm{v}$ formalin in PBS $(100 \mathrm{~mL}$ formalin (37-40\% stock solution), $900 \mathrm{~mL}$ Water, $4 \mathrm{~g} / \mathrm{L}$ $\mathrm{NaH}_{2} \mathrm{PO}_{4}$ (monobasic), $6.5 \mathrm{~g} / \mathrm{L} \mathrm{Na}_{2} \mathrm{HPO}_{4}$ (dibasic/anhydrous, and observed by CLSM (Olympus IX 81, Center Valley, PA USA).

Percent parasite inhibition in treated wells was calculated usinধ the following formula reported and published by ou associated group previously (10).

\section{$P I=100-T \times 100 / C$}

Where PI is the percentage inhibition, $\mathrm{T}$ the number of parasites in treated samples/100 macrophage nuclei and $\mathrm{C}$ the number of parasites in control samples/100 macrophage nuclei.

\section{Statistical analysis}

Results were expressed as mean \pm S.D. Three sets of experiments were performed. The data were statistically processed by one-way analysis of variance (GraphPad; Prism software program) followed by post-hoc Tukey's multiple comparison tests to determine the level of significance.
Differences were considered statistically significant at $\mathrm{P}<$ 0.05 .

\section{Results}

Preparation and In-vitro characterization

PCL-NPs formulations, containing combination of dual agents via double emulsion method were prepared. PCL-NPs were optimized for various parameters. These include drug content, sonication time and polymer content. As the concentration of drugs (AmB and DOX) was gradually lowered, particle size was decreased while percent drug entrapment was increased (Table 1). However, as the concentration of AmB and DOX was gradually lowered, the number of nanoparticles increased (Table 1). It may be attributed to the leaky membrane formations which may result in lower entrapped volume and hence lower size of the particles. This hypothesis however, needs confirmation from experimental studies (10). When concentration of $\mathrm{AmB}$ and DOX was used at 5 and $10 \mathrm{mg}$ respectively, PCL-NPs formulations were formed within range, average particle size measured was $236.7 \pm 0.04 \mathrm{~nm}$ and entrapment efficiency was recorded to be $82.1 \pm 1.39$ (AmB) and $75.20 \pm 0.14$ (DOX) for PCL-NPs and again only nanoparticles were observed. Formulation having $\mathrm{AmB}$ and DOX of $5 \mathrm{mg}$ and $10 \mathrm{mg}$ respectively (formulation PCL-NP4)

Table 1 Optimization of AmB and DOX (mg)/weight of PCL-NPs (mg)

\begin{tabular}{ccccc}
\hline $\begin{array}{c}\text { Formulation } \\
\text { code }\end{array}$ & $\begin{array}{c}\text { AmB } \\
\text { content } \\
(\mathbf{m g})\end{array}$ & $\begin{array}{c}\text { DOX } \\
\text { content } \\
(\mathbf{m g})\end{array}$ & $\begin{array}{c}\text { \% Entrapment } \\
\text { efficiency }\end{array}$ \\
\hline PCL-NP1 & 10 & 20 & $65.3 \pm 1.10$ & $62.8 \pm 0.12$ \\
\hline PCL-NP2 & 8 & 15 & $70.1 \pm 1.08$ & $68.11 \pm 0.16$ \\
\hline PCL-NP3 & 6 & 12 & $78.4 \pm 1.12$ & $70.18 \pm 0.11$ \\
\hline PCL-NP4 & 5 & 10 & $82.1 \pm 1.39$ & $75.20 \pm 0.14$ \\
\hline PCL-NP5 & 3 & 8 & $88.6 \pm 1.62$ & $80.12 \pm 0.07$ \\
$\begin{array}{l}\text { Total polymer used-50 mg, sonication time-15 min } \\
\text { *(mean } \pm \text { S.D) } \mathrm{n}=3\end{array}$ & & &
\end{tabular}

was considered to be optimum because for intravenous or intracardiac administration purpose, particle size should be below $1 \mathrm{~mm}$ to prevent formation of emboli in the blood vessels (11) and formulation PCL-NP4 is having particle size in this range. Formulations with optimum drug to polymer content were subjected to sonication for different time periods to optimize the sonication time. Fig 1 shows that as the sonication time was increased from 0 to $15 \mathrm{~min}$, average particle size was recorded to be decreased. Above $15 \mathrm{~min}$ (i.e. at 20 and 25 min sonication) particle size were recorded to be increased which may be attributed to the particle coalescence 
occurred as a result of the high kinetic energy of the particles and they might have localized in hepatocytes apart of macrophages, the target site (12). Formulations with optimum drug content (AmB and DOX) to polymer content and sonication time were subjected to polymer ratio. From this hypothesis, optimum sonication time was recorded to be 15 min, which gave particle size of $236.7 \pm 0.04 \mathrm{~nm}$. As the PCL content was increased from 50 to $200 \mathrm{mg}$, percent drug entrapment was also recorded to be increased (Fig. 2).

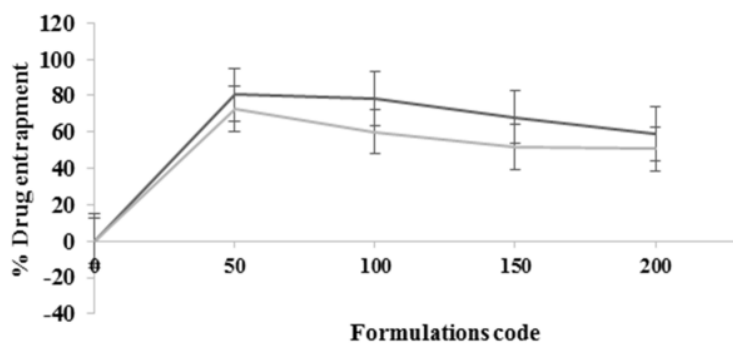

Figure 2 Optimization of PCL content in terms of \% drug entrapment and \% haemolysis. Total AmB-5 mg, DOX-10 $\mathrm{mg}$, sonication time- $15 \mathrm{~min}$, speed- 10,000 , Temp- $25{ }^{\circ} \mathrm{C}$

This may be attributed to the formation of thick layers upon which drugs were encapsulated (13). Also as the PCL content was increased, percent haemolysis was also recorded to be higher (Fig. 2). The mechanism responsible for this toxicity is not understood (14). However, it may be attributed to the subsequent decreased stability of the NPs with the increase in PCL content and initial fast release of drug from these unstable particles (15). Optimum PCL content was found to be $50 \mathrm{mg}$ (Fig. 2) which could entrap maximum amount of drug $82.1 \pm$ $1.39(\mathrm{AmB})$ and $75.20 \pm 0.14(\mathrm{DOX})$ for PCL-NPs with an acceptable level of toxicity towards erythrocytes.

After optimizing the process parameters, PCL-NPs were coated with macrophage specific ligand lectin $(18,19)$. Moreover, lectin possessed a positive charge, while PCL-NPs were negatively charged which further facilitated the adsorption process and resulted in a reduction of the zeta potential of the dispersion, which was used to optimize the process variables $(16,17)$. This determinant variable was critically utilized in determining optimal ligand density through optimization of process variables (polymer-to-lectin ratio and incubation time) (18). The increase in average particle size in case of lectin coated PCL-NPs (252.3 $\pm 0.04 \mathrm{~nm})$ as compared to plain PCL-NPs $(236.7 \pm 0.04 \mathrm{~nm})$ is an indication of coating. Zetapotential value declined steeply from their initial values, which might have been attributed to the charge quenching of the surface associated with lectin. With a longer incubation time (beyond $6 \mathrm{hr}$ ), the amount of residual free lectin was decreased and the change in zeta potential was not significant. This indicated that at the end of 6th hr, the interaction and inter-digitation of added lectin could have completed. The shape and morphology of the PCL-NPs were determined by TEM. The TEM photograph suggested that the PCL-NPs are spherical in shape and multilamellar in nature (Figure 6). The presence of coating on the PCL-NPs surface could also be appreciated from the TEM, which indicates surface intervening and anchoring of ligand (Figure 6). For PCL-NPs the initial negative value of the zeta potential was changed from -12.5 to 4.51 mVon addition of cationic ligand lectin and approached towards a minimum value (Fig. 3).

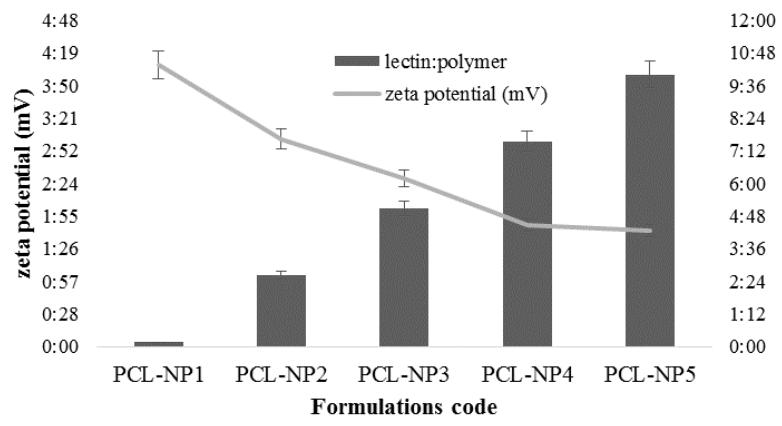

Figure 3 Optimization of lectin to polymer ratio for complete lectin coating over PCL-NPs. Incubation time of $6 \mathrm{hrs}$ was kept constant.

It was apparently related to the extent of the masking of the surface charge by the lectin. On further addition of lectin especially beyond this optimum ratio $(3: 1 \mathrm{w} / \mathrm{w})$ no significant change in the zeta potential was occurred for PCL-NPs. Hence optimum ratio of lectin: polymer was recorded to be $3: 1(\mathrm{w} / \mathrm{w})$ for PCL-NPs. This indicated that at an optimal 3:1 w/w ratio of lectin: polymer, the integration of lectin with the surfactant membrane was occurred at saturation level. For optimization of incubation time, the PCL-NPs formulations using the optimum lectin: polymer ratio were incubated with lectin for different time periods $(0,1,2,3,4,5$ and $6 \mathrm{~h})$ and the change in zeta potential was recorded (Fig. 4). Table 2 shows the average particle size and percent drug entrapment of optimized formulation of

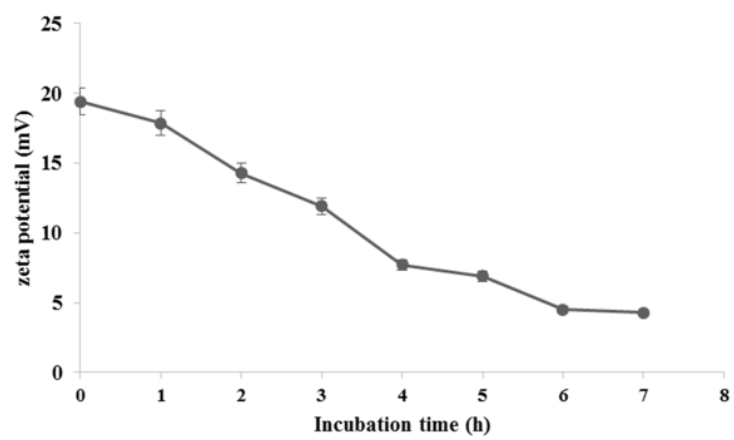

Figure 4 Optimization of incubation time for complete lectin coating over PCL-Nanoparticles. Lectin to polymer ratio 3:1 w/w was kept constant. 
Table 2 Characterization of optimized free and lectin coated PCL-NPs

\begin{tabular}{c|c|c|c|c}
\hline $\begin{array}{c}\text { Formulation } \\
\text { code }\end{array}$ & $\begin{array}{c}\text { of } \\
\text { of } \\
\text { ligand }\end{array}$ & Charge & $\begin{array}{c}\text { \% Drug } \\
\text { entrapment } \\
\text { AmB } \\
\text { DOX }\end{array}$ & $\begin{array}{c}\text { Average } \\
\text { particle } \\
\text { size (nm) }\end{array}$ \\
\hline PCL-NPs & ---- & Negative & $\begin{array}{c}82.1 \pm 1.39 \\
80.1 \pm 1.29\end{array}$ & $236.7 \pm 0.04$ \\
\hline Lectin-PCL- & lectin & Positive & $\begin{array}{c}75.20 \pm 0.14 \\
73.07 \pm 0.75\end{array}$ & $252.3 \pm 0.04$ \\
\hline NPs & & & & \\
\hline
\end{tabular}

$*($ Mean \pm S.D $) n=3$

PCL-NPs and lectin-PCL-NPs. The increase in average particle size in case of lectin-PCL-NPs as compared to PCLNPs is an indication of coating, which can be distinguished by dark black boundary of the formulation lectin-PCL-NPs. Also the change in zeta potential of the PCL-NPs indicates the presence of coating as previously zeta potential was negative and after coating it changes into positive. Percent drug entrapment of optimized PCL-NPs and lectin-PCL-NPs formulation was recorded to be $82.1 \pm 1.39(\mathrm{AmB})$ and 75.20 $\pm 0.14(\mathrm{DOX})$ and $80.1 \pm 1.29(\mathrm{AmB})$ and $73.07 \pm 0.75 \%$ (DOX) $(\mathrm{w} / \mathrm{w})$ respectively, revealing that lectin anchoring result in significant increase of the percent drug entrapment (Table 2). Preformed PCL-NPs were used for anchoring of ligand and this may presumably be the reason for the insignificant change recorded in the percent drug entrapment value (17). Relatively high entrapment of AmB and DOX in the PCL-NPs could be attributed to the hydrophobic nature of the drug, since the entrapment was dependent upon lipid: aqueous phase ratio.

\section{Ex-vivo stability}

Stability of developed PCL-NPs in serum was measured as percent drug leaching from PCL-NPs and changes in particle size of PCL-NPs after incubation with serum at $37 \pm 1^{\circ} \mathrm{C}$. The PCL-NPs were found to be almost constant upon incubation with freshly pooled rat serum (Figure 5). Only $6.6 \pm 0.35$ and $5.1 \pm 0.21 \%$ drug was leached into serum after $24 \mathrm{hr}$ of incubation from plain and lectin coated PCL-NPs, respectively. This may be attributed to the hydrophobic nature of the formulation hence prevented the drug leaching in serum. Results also indicate that lectin coating considerably reduced the leaching of the drug from PCL-NPs (12). Similarly, particle size analysis of PCL-NPs did not show any significant change in the particle size of plain and lectin coated PCL-NPs after $24 \mathrm{~h}$ incubation with serum (data not shown). There was only minor increase in the particle size of plain and lectin coated PCL-NPs.

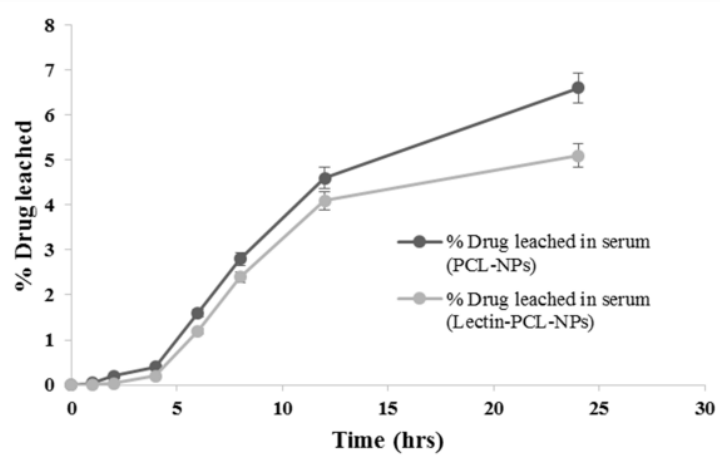

Figure $5 \mathrm{In}$-vitro drug leaching from plain and lectin coated PCL-NPs in serum after different time intervals

\section{In vitro phagocytic uptake studies in macrophage cell line} Qualitative analysis of in-vitro phagocytic uptake of PCL-NPs was studied using macrophage J774.1 cells in light microscopy (Figure 6). AmB and DOX loaded PCL-NPs were non-

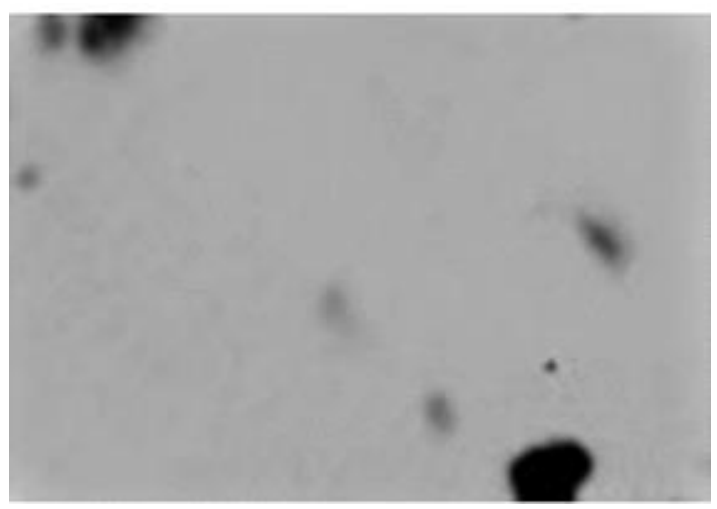

Figure 6 TEM image of lectin coated PCL-NPs (X 40000)

specifically taken up by macrophage cell lines and the maximum uptake was observed at $60 \mathrm{~min}$. Although some PCL-NPs were found distributed and attached to cell surface, active non-specific phagocytic uptake was noticed in most wells on close observation. Staining was carried out using Giemsa stain elsewhere in the body and its bioavailability towards spleen was nominal. Free nanoparticles alone caused in very low or negligible suppression due to its immune response. Ultimately, it was clearly clarified that an optimal quantity of $\mathrm{AmB}$ and $\mathrm{DOX}$ required is reaching spleen and liver via macrophage transport. A $74.2 \pm 1.9(\mathrm{AmB})$ and 70.52 \pm 1.2 (DOX) parasitic suppression indicates that our prepared delivery system can be a capable tool for anti-leishmanial therapy. 


\section{Discussion}

PCL-NPs have been recognized as potent drug delivery systems for the treatment of Visceral leishmaniasis (VL) because both nanoparticles as well as leishmania parasite are taken up by the same reticuloendothelial system (RES) and it creates an ideal situation for a high degree of drug parasite interaction (16). Furthermore, if suitable ligands are incorporated to PCL-NPs so that they could easily be recognized by the macrophages (host for the parasite) receptors, then these customized PCL-NPs could probably be used successfully as carriers for site specific delivery (17).The majority of the drugs used formerly in the therapy of visceral leishmaniasis were toxic. However when those drugs were Nanoparticles-encapsulated, they were found to be less toxic and more efficient in the therapy of visceral leishmaniasis (16). In the present study the efficacy of AmB and DOX in the form of novel carrier, PCL-NPs was tested against VL in macrophage cell line and compared with free AmB and DOX and indicates that prepared delivery system can be a capable tool for anti-leishmanial therapy. This may be attributed to the nano size range of PCL-NPs which forced the particles to accumulate in the macrophage rich organs like spleen, hence related to a different drug biodistribution in the form of carrier. Moreover formulation Lectin-PCL-NPs was found to be most potent as compared to PCL-NPs which includes involvement of mannose receptors expressed onto the membrane of macrophages of the liver and spleen. These receptors might have been involved in the selective and higher uptake of the ligand appended nanoparticles (lectin-PCL-NPs) due to ligandreceptor interaction (16).

\section{Conclusion}

We have proved the efficacy of AmB and DOX-loaded PCLNanoparticle (PCL-NPs) delivery system used for anti-VL using macrophage-targeted drug delivery approach. The nanoparticles of PCL were successfully prepared by double emulsion method with optimum size and entrapment. By exploiting passive targeting modes, total dose required for therapy was grossly cut down to few orders extent less than free drug, rendering the therapy favourable and affordable. The developed systems (plain and lectin coated PCL-NPs) via intravenous/ intracardiac administration appear promising for the treatment of VL specifically and hepato-splenic candidiasis. Developed formulation system exhibited no major side effects and thereby ensuring safer use over time and is possibly a better substitution for antimonials or other anti VL drugs which is suffering resistance and toxicity concerns. Further extensive studies on stability aspects should be carried out before proceeding to industrial scale-up.

\section{Acknowledgements}

Author Prachi Sharma is thankful to Apeejay Stya University, New Delhi, India, for providing necessary facilities for research work. The help and facilities provided by the Central Drug Research Institute, Lucknow (UP), India, are also duly acknowledged.

\section{Conflict of interest}

The authors report no conflicts of interest.

\section{References}

1. Nan A, Croft SL, Yardley V, Ghandehari H. Targetable watersoluble polymer-drug conjugates for the treatment of visceral leishmaniasis. J Control Release 2004;94:115-27.

2. Kumar N, Sharma P, Jaiswal A, Dube A, Gupta S Development and evaluation of paminophenylmannopyranoside anchored emulsomes for treatment of experimental visceral leishmaniasis. Ann Clin Cytol Pathol 2016;2:1042.

3. Khare P, Rastogi P, Gupta S, Maurya R, Dube A. In vitro and in vivo efficacy of a new herbaceous indian plantAbutilon indicum against Leishmania donovani infection. Amer J Phytomed Clin Ther 2014; 2:134-9.

4. Kumari S, Kumar A, Samant M, Singh N, Dube A. Discovery of novel vaccine candidates and drug targets against visceral leishmaniasis using proteomics and transcriptomics. Curr Drug Targets 2008;9:938-47.

5. Gupta S, Dube A, Vyas SP. Development and characterization of amphotericin B loaded solid lipid nanoparticles against experimental visceral leishmaniasis. Pharm Nanotech 2013;1:54-67

6. Pal A, Gupta S, Jaiswal A, Dube A, Vyas SP. Development and evaluation of tripalmitin emulsomes for the treatment of experimental visceral leishmaniasis. $\mathrm{J}$ Liposome Res 2012;22:62-71.

7. Mukherjee S, Das L, Kole L, Karmakar S, Datta N, Das PK. Targeting of parasite-specific immunoliposome-encapsulated doxorubicin in the treatment of experimental visceral leishmaniasis. J Infect Dis 2004;189:1024-34.

8. Kalaria DR, Sharma G, Beniwal V, Ravi Kumar MN. Design of Biodegradable Nanoparticles for Oral Delivery of Doxorubicin: In vivo Pharmacokinetics and Toxicity Studies in Rats. Pharm Res 2009;26:492-501.

9. Lammers T, Subr V, Ulbrich K, Peschke P, Huber PE, Hennink WE, et al. Simultaneous delivery of doxorubicin and gemcitabine to tumors in vivo using prototypic polymeric drug carriers. Biomaterials 2009;30:3466-75.

10. Vyas SP, Quraishi S, Gupta S, Jaganathan KS. Aerosolized liposome-based delivery of amphotericin B to alveolar macrophages. Int J Pharm 2005;296:12-25.

11. Kunjachan S, Gupta S, Dwivedi AK, Dube A, Chourasia, M. Chitosan-based macrophage-mediated drug targeting for the treatment of experimental visceral leishmaniasis. J microencapsul 2011;28:301-10.

12. Dubey N, Varshney R, Shukla J, Ganeshpurkar A, Hazari PP, Bandopadhaya GP, et al. Synthesis and evaluation of biodegradable PCL/PEG nanoparticles for neuroendocrine tumor targeted delivery of somatostatin analog. Drug Deliv 2012;19:132-42.

13. Sharma S, Kumar P, Jaiswal A, Dube A, Gupta S Development and characterization of doxorubicin loaded microparticles against experimental visceral leishmaniasis. J Biomed Nanotech 2011;7:135-6.

14. Italia JL, Bhatt DK, Bhardwaj V, Tikoo K, Kumar MN. PLGA nanoparticles for oral delivery of cyclosporine: nephrotoxicity and pharmacokinetic studies in comparison to Sandimmune Neoral. J Control Release 2007;119:197-206.

15. Roy $\mathrm{P}$, Das S, Bera T, Mondol S, Mukherjee A Andrographolide nanoparticles in leishmaniasis: 
characterization and in vitro evaluations. Int $\mathrm{J}$ Nanomedicine 2010;5:1113-21

16. Gupta S, Dube A, Vyas SP. Antileishmanial efficacy of amphotericin B bearing emulsomes against experimental visceral leishmaniasis. J Drug Target 2007;15:437-44.

17. Gupta S, Vyas SP. Development and characterization of amphotericin B bearing emulsomes for passive and active macrophage targeting. J Drug Target 2007;15:206-17.
18. Costa Lima SA, Resende M, Silvestre R, Tavares J, Ouaissi A, Lin PK, et al. Characterization and evaluation of BNIPDaoctloaded PLGA nanoparticles for visceral leishmaniasis: in vitro and in vivo studies. Nanomedicine 2012;7:1839-49.

19. Yin $\mathrm{Y}$, Chen D, Qiao M, Lu Z, Hu H. Preparation and evaluation of lectin-conjugated PLGA nanoparticles for oral delivery of thymopentin. J Control Release 2006;116: 337-45. 